Methods Views of hospital physicians caring for people with conditions causing chronic breathlessness were explored in a South Indian hospital as part of a service improvement project. Three semi-structured focus groups were conducted in English, audio-recorded, transcribed and subjected to thematic analysis.

Results Fifteen clinicians participated, representing oncology, palliative care, cardiology and respiratory specialties. Three major themes (Impact, Invisibility and Purpose) and 12 subthemes were generated by the data.

Chronic breathlessness as defined, was seen as prevalent with a huge impact on patients, their families, carers and clinicians. In non-palliative care clinicians, a sense of therapeutic helplessness with a lack of awareness or ability to manage chronic breathlessness was observed in association with active avoidance. This, a perceived lack of assessment tools and lack of clear clinical pathways allowing access to palliative care contributed to the invisibility of people with this symptom.

Most participants were in agreement about the name of chronic breathlessness syndrome. All agreed that focussed systematic identification would foster education regarding assessment, management and monitoring and would support service development and research.

Conclusions Chronic breathlessness syndrome is recognised in clinical practice in Southern India but risks being invisible due to lack of awareness, particularly of interventions to manage the breathlessness itself. A named and defined syndrome was seen as a way to improve identification and management of chronic breathlessness.

\section{THE SYMPTOM BURDEN AND QUALITY OF LIFE IN CANCER PATIENTS IN GAZA, PALESTINE: A CROSS- SECTIONAL STUDY}

Yousuf ElMokhallalati, Enas Alaloul, Mohammed Shatat, Tasneem Shneewra, Saad El Massri, Omar Shaer, Matthew Allsop. University of Leeds, Ministry of Health (Palestine), Islamic University (Palestine)

\subsection{6/spcare-2020-PCC.118}

Background Cancer is the second leading cause of death in Palestine. Cancer patients usually suffer high levels of physical and psychological symptoms. In Gaza, there are no studies assessing symptom burden and quality of life (QoL) using validated tools. The aim of this study was to assess symptom burden and QoL in a representative sample of cancer patients accessing outpatient services in the Gaza Strip.

Methods A cross-sectional, descriptive survey was used. The Lebanese version of the Memorial Symptom Assessment Scale (MSAS-Leb) and the Arabic version of the European Organization for Research and Treatment of Cancer Quality of Life Questionnaire-C30 (EORTC QLQ-C30) were used to collect data from cancer patients accessing oncology outpatient services at Al Rantisi Hospital or European Gaza Hospital (EGH) from July to August 2019. Analysis is ongoing including modelling to derive determinants of symptom prevalence and QoL.

Results Data were collected from 385 cancer patients (93\% response rate) at $\mathrm{Al}$ Rantisi Hospital and EGH. The majority of participants were women with breast cancer and the mean age was 52 years. The most commonly reported physical symptoms were lack of energy and pain and the most common psychological symptoms were feeling nervous and feeling sad. A higher level of burden of global distress, physical, and psychological symptoms was found in patients categorised as either 'Divorced/Widowed/Widower', with less than secondary school education or low income. The physical and role functioning domains of the EORTC QLQ-C30 were found to have the lowest score and cognitive functioning the highest. Conclusions A high symptom burden was identified across participants. There remain many unresolved problematic symptoms for patients with cancer that affect their QoL. Utilising such systematic assessment of symptom burden and QoL can help to inform guidance and protocols for treatment and follow up of cancer patients in this context.

\section{THE ROLE OF CIVIL SOCIETY IN THE PROMOTION OF PALLIATIVE CARE: CASE OF PALLIATIVE CARE ASSOCIATION OF RWANDA (PCAR)}

Rose Gahire, Grace Mukankuranga. Palliative Care Association of Rwanda (APCAR)

\subsection{6/spcare-2020-PCC.119}

The concept of Palliative care in Rwanda was initiated in 2004, after realization of the need for Palliative care services for the patients with chronic illnesses like cancer and HIV/ AIDS. The 1st Palliative Care introduction course was held for health professionals from hospitals and NGOs in 2006.

The role of PCAR in promotion/Provision of PC in Rwanda; 1) Advocacy :(Policy level)

Contributed to the development of Rwanda National Stand -alone palliative care policy, development of standard and guidelines on PC, development of Five year National strategic and implementation plan on PC, availability and accessibility of strong opioids and for integration of PC at all levels of health care. 2)Capacity strengthening; trained 168 trainers (TOTs) of health professional in referral and District Hospitals, 250 Health care professional trained from from different Hospitals and 100 Community health workers. 3) Service Delivery: Started a Hospice and Home palliative care and reached 120 patients, provided to 88 patients: comfort fund, Bereavement support and home based care.

Key Results Government integrated PC into all levels of Health system, Availability of Syrup Morphine, was put on essential drugs list, revision of law concerning the use of Narcotic drugs (allowing Nurses to prescribe Morphine)

Way forward Establish partnership between churches, Rwanda Correctional Service (prisons), and private hospitals.High-level lobbying: government institutions to understand PC (Parliament), Resource mobilization both inside and outside the country. Continuous in-service training on PC, Annual Stakeholders meeting to monitor the implementation of national PC policy, and scale up PC services especially home and hospice care.

\section{HOW DO COMMUNITY-BASED PALLIATIVE CARE PATIENTS IN THE USA VIEW EMERGENCY HEALTH CARE PLANS?}

L Srivastava, R Krueger, B Rose. PalliaCare Cincinnati, Hospice of Cincinnati, Trihealth

\subsection{6/spcare-2020-PCC.120}

Background Advance care planning (ACP) improves the quality of life, reduces hospital admissions and increases choice for patients with life limiting conditions. ACP includes advance statements, health care power of attorney and emergency 